\title{
INTELLIGENT TRAFFIC REGULATION SYSTEM FOR ROADS USING CAR TWO WAY COMMUNICATION
}

\author{
Bestley Joe. $\mathbf{S}^{\mathbf{1}}$, Vamsi Krishna. $\mathbf{P}^{\mathbf{2}}$ \\ ${ }^{1}$ Assistant Professor, Department of Electronics \& Instrumentation, Sathyabama University, Tamil Nadu, India \\ ${ }^{2}$ Assistant Professor, Department of Electronics \& Control, Sathyabama University, Tamil Nadu, India
}

\begin{abstract}
A Car-2-X communication system using RF technology would demonstrate the usefulness of this technology which would be applied for, advanced driver assistance increasing road safety by reducing the number of accidents as well as reducing the impact in case of non-avoidable accidents, increasing traffic efficiency with traffic congestion control resulting in reduced transport time, fuel consumption and thus contributing to improving the environment, user communications and information services offering comfort and business applications to driver and passengers.
\end{abstract}

Keywords: RF technology, 2-X communication system, traffic efficiency, congestion control.

\section{INTRODUCTION}

Car2x communication (also known as Vehicle-to-Vehicle and Vehicle-to Infrastructure communication) is the exchange of information between traffic participants and the infrastructure with the goal of enhancing safety and convenience and optimizing traffic flow. The recent engineering system for assuring Car $2 \mathrm{x}$ communication is known as the intelligent transport System (ITS). The basic concept of Car2x communication involves sending and receiving standardized messages over the air interface and enabling interpretation of the status information they contain by traffic participants. The ITS station (ITS-S) keeps the messages up-to date based on the momentary traffic situation and sends them either periodically or they are event-driven.

The most important status information is transmitted via the message types CAM(Cooperative Awareness Message), SPAT (Signal Phase and Time), DENM (Decentralized Environmental Notification Message) and TOPO (Topology Specification). The European Institute for Telecommunication Standards (ETSI) has already specified the CAM and DENM messages whereas SPAT and TOPO are currently handled on a project-by-project basis. This system gives the intelligent processing units of the receiving traffic participants, e.g. in a vehicle, the opportunity to acquire information about the immediately relevant traffic situation over a broad area and to warn the vehicle driver if necessary or even intervene in vehicle control.

\section{EXISTING SYSTEM}

The rapid growth in the vehicle ownership is one of the measures for economic growth of country. Exploitation of new trends and technologies requires fast transportation of all goods alike.
The goal of each one is to reach at destination without wasting time and money. But the infrastructures provided by current resources are very limited. So the traffic management at road is crucial to reduce waiting and travelling times, save fuel and money.

In current scenario, the low or high traffic information is offered by only who are affected by that traffic problem for waiting long time to get signal to move other side.

\section{DISADVANTAGES OF EXISTING SYSTEM}

1) Waiting too long time for getting the traffic signal.

2) Wastage of fuel.

3) If accidents occur, sharing the information is taking much amount of time.

\section{PROPOSED SYSTEM}

The project will implement and demonstrate the following use cases.

Forward Hazard Warning: If a vehicle detects an incident such as emergency braking, accident, traffic jam, slippery road, or construction zone, it adds this information to its periodically broadcasted Cooperative Awareness Message which is sent to all vehicles in the close vicinity. Other cars or motorcycles receives the information and decide either to warn the driver if the hazardous location is on the route in front or just to broadcast the information to warn other vehicles.

Green Light Optimal Speed Advisory: The vehicle approaches the traffic light that is currently red. Based on the received traffic light phase schedule, the vehicle calculates an approaching speed of say, $45 \mathrm{~km} / \mathrm{h}$, at which the vehicle would reach the traffic light at the beginning of the next green phase. This information is presented to the driver who can avoid the unnecessary stop. If the vehicle is 
somehow needed to stop, then the traffic light would automatically switches off the car engine and later switch ON the ignition once it shows green.

Road Speed Limit Warning: The road speed limit warning application allows connected vehicles to receive information that it is approaching a road along with the recommended speed for the curve. This allows the vehicle to provide a warning to the driver regarding the road and its recommended speed. In addition, if the actual speed through the curve exceeds the recommended speed, the vehicle can communicate and informs this to the traffic control unit, where the vehicle number will be displayed. If the driver drives the vehicle faster than the speed limit ignoring the warning, the vehicle unit would inform the vehicle details to the traffic control unit.

Emergency Vehicle Warning: While the vehicles are driving along the road an emergency vehicle approaches from behind. This system uses information from the EV to help the driver on how to clear the road for the emergency vehicle even when the siren and light bar may not yet be audible or visible. The driver/rider of the other vehicle will be advised to stop at the road side to let the EV pass by. If the driver doesn't slow the vehicle after this warning, the vehicle details will be automatically transferred to the traffic control unit.

The project consists of three units running a wireless network among them. The control and monitoring unit has a touch screen display and other sensors. Vehicle units have a compass, a graphics LCD and a warning buzzer.

\section{ADVANTAGES OF PROPOSED SYSTEM}

Safety

Traffic Management

Driver Assistance System

Policing and Enforcement

\section{TRAFFIC MODULE}

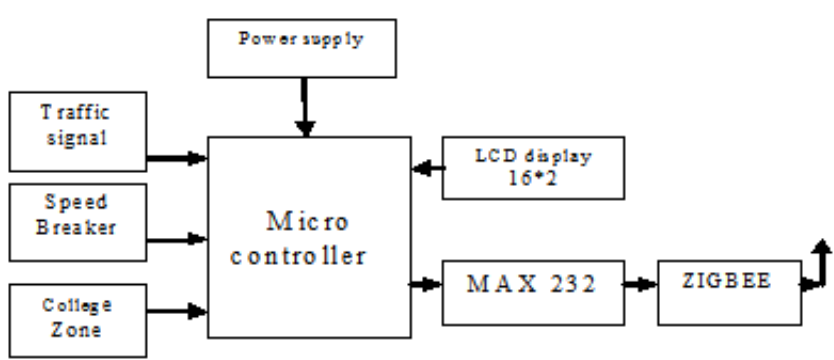

Fig -1: Block Diagram of Traffic Module

Fig -1 represents the block diagram of traffic module. The power supply is given to the microcontroller used in the traffic module .The microcontroller starts its process. The traffic display (Light Emitting Diode) shows the signal and the signal time variations are displayed in LCD display. The information is passed from the microcontroller to zigbee through an interface $\max 232$ connected in between microcontroller and zigbee. The message is being passed to car which is a head towards the signal. The purpose of two IR sensors is to measure the traffic density, speed breakers and the college zones and then the IR sensors sends the information to microcontroller and the information is transmitted through the zigbee.

\section{CAR 1 AND CAR 2 MODULE}

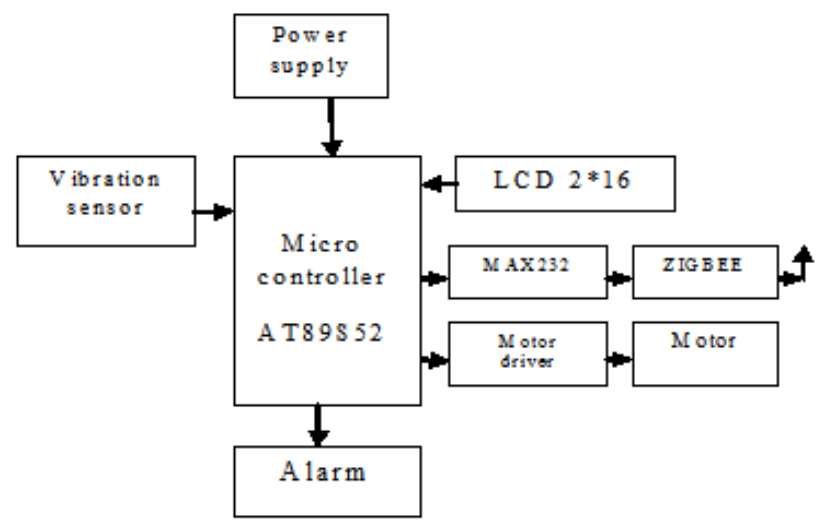

Fig -2: Block Diagram of Car 1 Module

Figure: 2. which represent the block diagram of car1 module. The block diagram shows the working of car1 module. The process is dumped in the microcontroller through programmer. In carlmodule the zigbee used for both transmitting and receiving signal from traffic module and emergency module. The zigbee is interfaced to the microcontroller through max 232 used as an interface. The Zigbee receives the signal from traffic module and transmits signal to car2 module. The vibration sensor used in this module is to sense when the car gets crashed and starts to vibrate were the vibration signal is been passed as the electrical signal through the vibration sensor and the microcontroller sends the signal to other vehicles. The motor driver is used for controlling the speed of the vehicle.

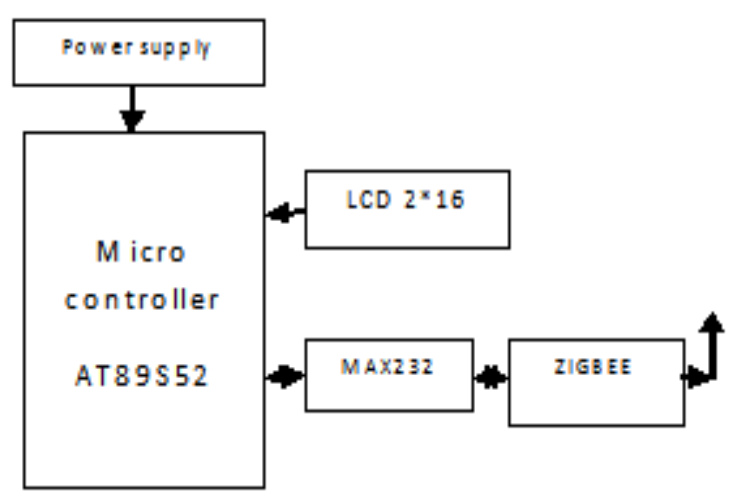

Fig -3: Block Diagram of Car 2 Module.

Figure:3 represent the block diagram of car2 module. The block diagram shows the working of car2 module. The car2 module consists of microcontroller ,LCD, $\max 232$, Zigbee, and power supply. The car2 module receives the signal from car1 module, and from the emergency module. The receiving signal is displayed in the $16 * 2$ LCD display. 


\section{EMERGENCY MODULE}

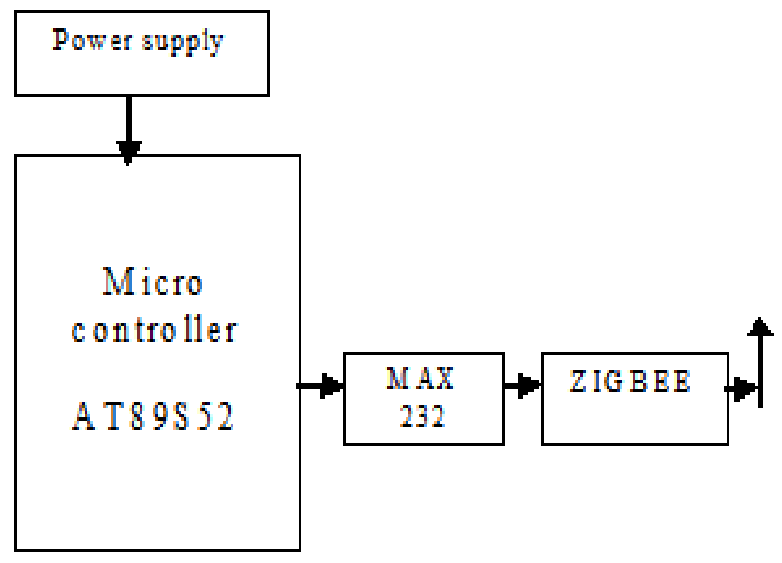

Fig -4: Block Diagram of Emergency Module.

Figure: 4. represent the block diagram of emergency module. The emergency module operates when the power supply is given to microcontroller. The microcontroller passes information as signal through zigbee to the vehicles

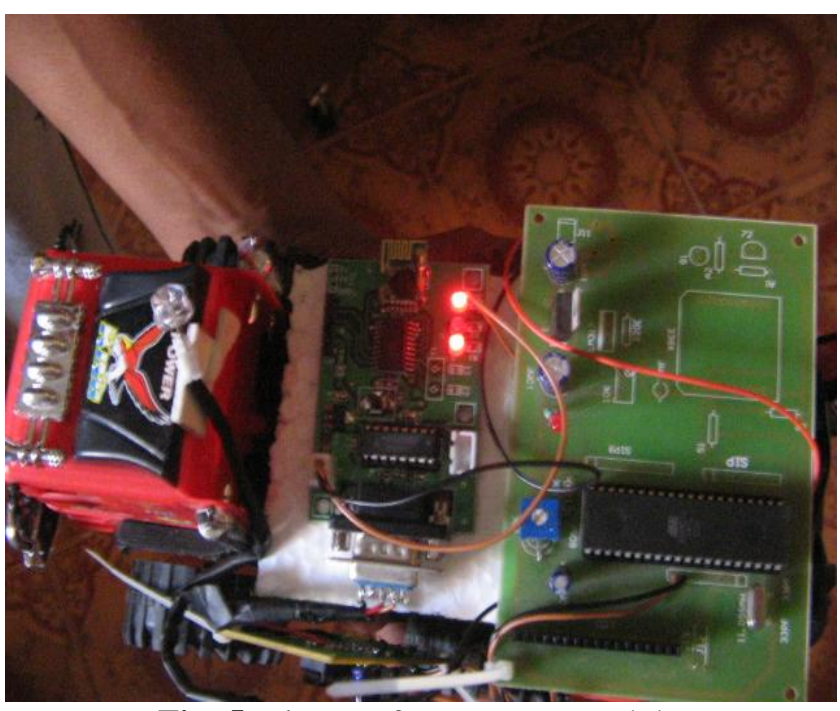

Fig -5: Picture of Emergency module

\section{SUMMARY AND CONCLUSIONS}

This report shows the complete working of car-2-x communication systems. The various hardware and software used in this project is thoroughly seen along with their working principle. In this paper we have successfully concluded the zigbee based communication using keil micro version.

Thus in the end of the process the supervisor could see the visual output of the process via zigbee and he could in-turn control the process similarly the head person of the process could get the output of the process in abnormality condition. Thus the whole project is been executed successfully and the desired result of road link car 2-X communication sytems is obtained.

\section{FUTURE WORK}

The present demo car in which was successfully implemented the 2 way communication to reduce congestion, traffic density, accidents and emergency situation can be extended in full real life road situation and also if we were provided with proper hardware we can fully automate it.

\section{REFERENCES}

[1]. Kaltwasser J, Kassubek.J, "A new cooperative optimized channel access for inter-vehicle communication", vehicle navigation and information systems conference, 1994.

[2]. Timo Sukuvara, Pertti Nurmi, Daria Stepanova, Sami Suopajarvi, Marjo Hippi, "Wireless Traffic Service Communication platform for cars", $2^{\text {nd }}$ IEEE workshop on automatic networking \& applications (Autonet 2007), November 2007

[3]. Piao.J, Mc Donald M, "Potential applications of roadvehicle communication for improving safety and mobility", in Road transport information \& control-RTIC 2008 and ITS United Kingdom members conference.

[4]. A.C.Brown, E.J.Cullen, M.Brackston, D.J.Gunton, "Vehicle to Vehicle communication outage \& its impact on convoy driving", communication \& Information systems Division, BAE systems advanced technology centres.

[5]. Kenneth J Ayala, "The 8051 Microcontroller Architecture, Programming \& Applications", Penram International, 2nd Edition, 1996. Page no. 22-42, 51-83, 109-122, 543-550

[6]. Muhammad Ali Mazidi, Janice Gillispie Mazidi, Rolin D. Mc Kinlay , "The 8051Microcontroller \& Embedded System", Pearson Education Inc. 2nd Edition, 2008. Page no. 13-151, 265-273, 289-292. 